\title{
Putative PPAR Target Genes Express Highly in Skeletal Muscle of Insulin-Resistant MetS Model SHR/NDmc-cp Rats
}

\author{
Natsuyo Hariya ${ }^{1,2}$, Kunio MiYAKE ${ }^{2}$, Takeo KubOTA ${ }^{2}$, \\ Toshinao GODA ${ }^{3}$ and Kazuki MochIZUKI ${ }^{3,4, *}$ \\ ${ }^{1}$ Interdisciplinary Graduate School of Medicine and Engineering, University of Yamanashi, 4-4-37 Takeda, \\ Kofu, Yamanashi 400-8510, Japan \\ ${ }^{2}$ Department of Epigenetic Medicine, Interdisciplinary Graduate School of Medicine and Engineering, \\ University of Yamanashi, 4-4-37 Takeda, Kofu, Yamanashi 400-8510, Japan \\ ${ }^{3}$ School of Food and Nutritional Sciences, University of Shizuoka, 52-1 Yada, Suruga-ku, \\ Shizuoka 422-8526, Japan \\ ${ }^{4}$ Laboratory of Food and Nutritional Sciences, Department of Local Produce and Food Sciences, \\ Faculty of Life and Environmental Sciences, University of Yamanashi, 4-4-37 Takeda, Kofu, \\ Yamanashi 400-8510, Japan \\ (Received April 25, 2014)
}

\begin{abstract}
Summary It is known that insulin resistance in skeletal muscle induces subsequent metabolic diseases such as metabolic syndrome (MetS). However, which genes are altered in the skeletal muscle by development of insulin resistance in animal models has not been examined. In this study, we performed microarray and subsequent real-time RT-PCR analyses using total RNA extracted from the gastrocnemius muscle of the MetS model, spontaneously hypertensive corpulent congenic (SHR/NDmc-cp) rats, and control Wistar Kyoto (WKY) rats. SHR/NDmc-cp rats displayed overt insulin resistance relative to WKY rats. The expression of many genes related to fatty acid oxidation was higher in SHR/NDmc-cp rats than in WKY rats. Among 18 upregulated genes, putative peroxisome proliferator responsive elements were found in the upstream region of 15 genes. The protein expression of ACOX2, an upregulated gene, and peroxisome proliferator-activated receptor (PPAR) G1, but not of PPARG2, PPARA or PPARD, was higher in the gastrocnemius muscle of SHR/NDmc-cp rats than that in WKY rats. These results suggest that insulin resistance in the MetS model, SHR/ NDmc-cp rats, is positively associated with the expression of fatty acid oxidation-related genes, which are presumably PPARs' targets, in skeletal muscle.
\end{abstract}

Key Words insulin resistance, skeletal muscle, fatty acid oxidation, PPAR, SHR/NDmc-cp rats

Skeletal muscle is responsible for approximately $75 \%$ of insulin-dependent glucose incorporation. A reduction in skeletal muscle glucose incorporation leads to the development of insulin resistance and subsequent development of metabolic diseases such as type 2 diabetes (1). In particular, insulin resistance with obesity in humans is frequently associated with hypertension and hyperlipidemia, as well as with hyperglycemia. Abnormal values of more than two of the following parameters-fasting

*To whom correspondence should be addressed.

E-mail:mochizukik@yamanashi.ac.jp

Abbreviations: Acox1, acyl-Coenzyme A oxidase 1, palmitoyl; Acsl1, acyl-CoA synthetase long-chain family member 1; ChIP, chromatin immunoprecipitation; CPT1B, carnitine palmitoyltransferase-1b; CVD, cardiovascular diseases; Cycs, cytochrome c, somatic; GLUT, glucose transporter; MetS, metabolic syndrome; PPAR, peroxisome proliferator-activated receptor; PPARGC1A, peroxisome proliferator-activated receptor (PPAR) $\gamma$ co-activator- $1 \alpha$; PPRE, peroxisome proliferator response elements; SHR/NDmc-cp, spontaneously hypertensive corpulent congenic; WKY, Wistar Kyoto; ZDF, Zucker diabetic fatty. blood glucose, lipids and blood pressure-in combination with obesity is generally called metabolic syndrome (MetS). It is known that subjects with MetS have subsequent higher incidences of cardiovascular diseases (CVD) and associated death (2). Therefore, maintaining insulin sensitivity in skeletal muscle by controlling dietary habits is important for preventing the onset and progression of MetS and related diseases.

The main mechanism of glucose uptake in skeletal muscle is via glucose transporter (GLUT) 4, which is translocated from intracellular storage vesicles to the plasma membrane in an insulin-dependent manner (3). Insulin-induced translocation of GLUT4 is signaled by the insulin receptor substrate-phosphoinositide 3-kinase pathway and by the AMP activated protein kinase pathway (4). Skeletal muscle glucose is stored as glycogen by dephosphorylated glycogen synthase via the insulin signal. These findings indicate that the insulin signal in skeletal muscle enhances glucose incorporation independently of expression changes in metabolic genes such as glycogen synthesis- and fatty acid synthesisrelated genes. 
Recently, many studies have demonstrated that fatty acid oxidation in the skeletal muscle is associated with insulin resistance in this muscle. Indeed, it has been reported in a human study that physical activity by subjects with moderate insulin resistance ameliorated the insulin resistance and reduced the expression of genes related to fatty acid oxidation including carnitine palmitoyltransferase- $1 \mathrm{~b}($ CPT1B) and peroxisome proliferator-activated receptor (PPAR) $\gamma$ co-activator- $1 \alpha$ (PPARGC1A, generally called PGC- $1 \alpha$ ) in skeletal muscle (5). Furthermore, a recent human study has demonstrated that the expression of fatty acid oxidationrelated genes such as PPARGC1A, CPT1B and PPARA (generally called PPAR $\alpha$ ), which is one of the nuclear receptors that activate fatty acid oxidation, in MetS subjects was positively associated with moderate insulin resistance, but not with severe insulin resistance (6). It is very likely that moderate insulin resistance induces fatty acid oxidation in skeletal muscle using fatty acids as an energy source. In contrast, development of severe insulin resistance and associated type 2 diabetes from moderate insulin resistance may suppress the fatty acid oxidation-related genes. In animal studies, it has been reported that insulin-resistant Zucker diabetic fatty (ZDF) rats, which unlike humans rapidly develop type 2 diabetes with pronounced hyperglycemia, did not show higher expression of genes related to fatty acid oxidation compared with the control animals $(7,8)$. In addition, an 8-wk high-fat diet intake induced moderate insulin resistance without changes in fatty acid oxidation-related genes in skeletal muscle of C57BL/6 J mice (9). These animal studies' results do not correlate with the human studies. Whether development of insulin resistance is associated with experimental changes in a series of fatty acid oxidation-related genes as well as other genes in skeletal muscle in models resembling humans should be examined.

In the present study, we examined by microarray analysis whether the expression of genes related to fatty acid oxidation as well as other genes in skeletal muscle differed between spontaneously hypertensive corpulent congenic (SHR/NDmc-cp) rats, an animal model of development of MetS with obesity, hyperlipidemia, hyperglycemia and hypertension based on insulin resistance accompanied by excessive eating (10), and the normal model, Wistar Kyoto (WKY) rats. Furthermore, to investigate whether the upregulated genes are regulated in the same fashion, we searched for putative peroxisome proliferator response elements (PPREs), which are important transcription factors for regulating fatty acid oxidation and other fatty acid metabolism (11), in the promoter/enhancer region of upregulated genes in skeletal muscle of SHR/NDmc-cp rats.

\section{MATERIALS AND METHODS}

Animals. Five-week-old male SHR/NDmc-cp rats $(n=6)$ and WKY rats $(n=5)$, which are control rats $(10$, 12), were purchased from Japan SLC, Inc. (Shizuoka, Japan) and maintained in cages under controlled conditions (temperature $23 \pm 2^{\circ} \mathrm{C}$; humidity $55 \pm 5 \%$; 12 -h light/dark cycle). Rats were fed a laboratory diet (MF; Oriental Yeast Co., Ltd., Tokyo, Japan) and had free access to food and water throughout the study. Nonfasting plasma samples at $18 \mathrm{wk}$ of age were collected from the tail vein using a capillary tube containing heparin-lithium (Terumo, Co., Ltd., Tokyo, Japan) between 9:30 am and 10:00 am. Rats were then killed by decapitation between 10:00 am and 12:00 pm, and gastrocnemius muscles were collected (13). The experimental procedures used in the study conformed to the guidelines of the Animal Usage Committee of the University of Shizuoka.

Microarray analysis. Total RNA was extracted by the acidified guanidine thiocyanate method, as described by Chomczynski and Sacchi (14). For microarray analysis, we used total RNA from three animals of each group. Total RNA (300 ng) was converted to complementary RNA (cRNA) and labeled with biotin using an Ambion WT Expression kit (Life Technologies, Carlsbad, CA), a GeneChip WT terminal labeling kit and an Eukaryotic poly-A RNA Control kit (Affymetrix, Santa Clara, CA) according to the manufacturers' instructions. The biotin-labeled cRNA $(10 \mu \mathrm{g})$ was hybridized to Rat 1.0 ST arrays (Affymetrix), washed and analyzed with GeneChip Scanner 3000 (Affymetrix) as previously reported (15). Variable spots detected by an algorithm in the Partec software (Ryoka System, Tokyo, Japan) in all plates were defined as non-expressed genes and were removed. The signals were normalized by the global median normalization method based on the Partec algorithm. Data are represented in base 2 logarithms $\left(\log _{2}\right)$, and only the signals in the microarray with differences between two groups that were greater than two after the $\log _{2}$ transformation are mentioned. The upregulated or downregulated genes with significant differences between the two groups, as calculated by Student's $t$-test $(p<0.05)$, were subjected to further analyses. Among the 1,477 gene signals detected in the microarray, we detected 42 upregulated genes and 33 downregulated genes in the SHR/NDmc-cp rats compared with the WKY rats. We focused on the upregulated genes, which were classified into nine groups based on their functions, namely energy/metabolism, signal transduction, transcription/chromatin, structural proteins, immune response, transport of nutrients/ions, stress, others, and unknown. Each gene was classified under a category based on gene ontology (GO) annotations and PubMed research. The GO annotations and the PubMed ID for each gene are indicated in Table 3. Genes related to nutrient metabolism were classified into the energy/ metabolism category. Genes related to G-protein coupled receptors, membrane receptors and cascades of phosphorylation signals under these receptors were classified into the signal transduction category. Transcriptional factors and transcriptional co-factors were classified into the transcription/chromatin category. Genes related to transport of nutrients and ions into the cells were classified into the transport of nutrients/ions category. Genes related to elimination of reactive oxygen species and oxidized substances were classified into the stress category. 
Table 1. Sequences of oligonucleotide primers used for real-time RT-PCR.

\begin{tabular}{|c|c|}
\hline Gene & Sequence \\
\hline Acyl-CoA oxidase 2 , branched chain $(A \operatorname{cox} 2)$ & $\begin{array}{l}\text { 5'-TGTGAGCTATGCCTTCCACTT-3' } \\
\text { 5'-GCCTTCATACCAGTGCTCAAT-3' }\end{array}$ \\
\hline Asparagine synthetase (glutamine-hydrolyzing) (Asns) & $\begin{array}{l}\text { 5'-TTCTGGCTGTGTGTTCAGAAG-3' } \\
\text { 5'-GGGCTCCACTTTTAGGAAGG-3' }\end{array}$ \\
\hline Acyl-CoA synthetase medium-chain family member 5 (Acsm5) & $\begin{array}{l}\text { 5'-CTTGCGTTTTTGTGCATGAG-3' } \\
\text { 5'-TTATTGGGAACCTGCAGAGAG-3' }\end{array}$ \\
\hline Pantothenate kinase 2 (Pank2) & $\begin{array}{l}\text { 5'-GACCTTGCCAGAGCAACTTT-3' } \\
\text { 5'-TTGCCGACAAATACAACCTG-3' }\end{array}$ \\
\hline Acyl-CoA thioesterase $2(A \cot 2)$ & $\begin{array}{l}\text { 5'-TTGAAGAAGCCGTGAACTACC-3' } \\
\text { 5'-GCCATTGATGACAACAGCAG-3' }\end{array}$ \\
\hline Phosphoserine aminotransferase 1 (Psat1) & $\begin{array}{l}\text { 5'-GGAATGGATCAAGAACAATGG-3' } \\
\text { 5'-CGGAATGTTCATCCTGCTTC-3' }\end{array}$ \\
\hline Nudix (nucleoside diphosphate linked moiety X)-type motif 7 (Nudt7) & $\begin{array}{l}\text { 5'-TCCCACTTGTCCCCTAGTAAGT-3' } \\
\text { 5'-CAGGTGCCCTTCTCAGCTT-3' }\end{array}$ \\
\hline GDP-D-glucose phosphorylase 1 (RDG1560277) & $\begin{array}{l}\text { 5'-CTAGGATATCCAACTCAGGTTGC-3' } \\
\text { 5'-CGTAGACAAAGTCGGGGATG-3' }\end{array}$ \\
\hline Melanocortin 5 receptor $(M c 5 r)$ & $\begin{array}{l}\text { 5'-TTCGACTCCATGATCTGCAT-3' } \\
\text { 5'-ATGTACCTGTCCACCGCAAT-3' }\end{array}$ \\
\hline $\begin{array}{l}\text { Phospholipase A2, group VII (platelet-activating factor acetylhydrolase, } \\
\text { plasma) (Pla } 2 g 7)\end{array}$ & $\begin{array}{l}\text { 5'-TCAAGCCCTAAGTGAAGACCA-3' } \\
\text { 5'-TGGAATTCGGCAGAGTTGAT-3' }\end{array}$ \\
\hline Activating transcription factor 5 (Atf5) & $\begin{array}{l}\text { 5'-CTGCTCACACCGACTCTTCA-3' } \\
\text { 5'-GTGAAGACAGGCACCAAGG-3' }\end{array}$ \\
\hline Smooth muscle alpha-actin (Acta2) & $\begin{array}{l}\text { 5'-CTCCCAGCACCATGAAGATT-3' } \\
\text { 5'-TCCACATTTGTTGGAAGGTAGAC-3' }\end{array}$ \\
\hline Interferon-induced protein with tetratricopeptide repeats 1 (Ifit1) & $\begin{array}{l}\text { 5'-CTTTGCTGAAATGCCACGTA-3' } \\
\text { 5'-GGATCACGAGAGCCATAAAGA-3' }\end{array}$ \\
\hline Catalase (Cat) & $\begin{array}{l}\text { 5'-TTCATCAGGGATGCCATGT-3' } \\
\text { 5'-TGATGGAGAGACTCTGGACAAA-3' }\end{array}$ \\
\hline Glycoprotein, alpha-galactosyltransferase 1,3 (Ggta1p) & $\begin{array}{l}\text { 5'-GCTCTGGGACTGGTTCAATC-3' } \\
\text { 5'-AAGTGCCTTCCCACACAATC-3' }\end{array}$ \\
\hline Receptor (chemosensory) transporter protein 4 (Rtp4) & $\begin{array}{l}\text { 5'-GGACCCTGCACTTGGATAAG-3' } \\
\text { 5'-CATCTGGAACACTGGAACCTG-3' }\end{array}$ \\
\hline Similar to interferon-inducible GTPase (RGD1309362) & $\begin{array}{l}\text { 5'-TACACCGCTCAATGTTGCTG-3' } \\
\text { 5'-TCTCCACCACCCCAATTTTA-3' }\end{array}$ \\
\hline Similar to hypothetical protein MGC29761 (RGD1306233) & $\begin{array}{l}\text { 5'-AAGAAAACCCCCAAGAGTGC-3' } \\
\text { 5'-TTTCTCTGGGGCAGGCTTA-3' }\end{array}$ \\
\hline General transcription factor IIB $(G t f 2 b)$ & $\begin{array}{l}\text { 5'-TACAGGAGCGGCAAGTTTTGA-3' } \\
\text { 5'-ATTGCGTGGGAGGTTGATTCT-3' }\end{array}$ \\
\hline
\end{tabular}


RNA analysis. Total RNA samples (250 ng) from SHR/NDmc-cp rats $(n=6)$ and WKY rats $(n=5)$ were converted into cDNA by reverse transcription (RT) using SuperScript ${ }^{\mathrm{TM}}$ III reverse transcriptase (Life Technologies) according to the manufacturer's instructions. To quantitatively estimate mRNA levels, PCR amplification was performed on the Light Cycler 480 System (Roche Diagnostics, Basel, Switzerland) as described previously (15). The primer sequences used are listed in Table 1.

Western blot analysis. Total protein was extracted from the gastrocnemius muscle using RIPA buffer and the soluble supernatants were then separated by $10 \%$ sodium dodecyl sulfate-polyacrylamide gel electrophoresis. Western blot analysis was performed as previously reported (13). The primary antibodies were A-TUBULIN (Sigma-Aldrich, St. Louis, MO), ACOX2 (Proteintech Group, Inc., Chicago, USA), PPARA (Santa Cruz Biotechnology, Santa Cruz, CA), PPARD (generally called $\operatorname{PPAR} \delta$; Santa Cruz Biotechnology) and PPARG (generally called PPAR $\gamma$; Cell Signaling Technology). Positive signals were detected by chemiluminescence (Western Lightning ECL Pro; PerkinElmer, Waltham, MA) using a Chemidog XRS plus instrument (Bio-Rad, Hercules, CA).

Searching for putative PPREs in the upstream region of the upregulated genes. Putative PPREs in the $-5000 \mathrm{bp}$ to $-1 \mathrm{bp}$ promoter/enhancer region of upregulated genes were searched for with the web program PPRESearch (http://www.classicrus.com/PPRE/) (16, 17). Search criteria were two mismatches, $80 \%$ binding efficiency, one directed repeat and zero flanking match. We searched both sense and antisense sequences.

Statistical analysis. Data are expressed as mean \pm standard error of the mean (SE). Significant differences between two groups were analyzed by Student's $t$-test. Values of $p<0.05$ were considered statistically significant.

\section{RESULTS}

Body weight and blood biochemical parameters in WKY and SHR/NDmc-cp rats

We previously reported the body weight and concentrations of blood glucose, insulin and triacylglycerol in SHR/NDmc-cp rats, WKY rats and streptozotocintreated rats (13). In this study, we now reanalyze the results from SHR/NDmc-cp rats and WKY rats using Student's t-test (Table 2).

Gene expression profiles of gastrocnemius muscles of WKY and SHR/NDmc-cp rats

We performed microarray analysis of total RNA from gastrocnemius muscles of WKY and SHR/NDmc-cp rats. We identified 14 upregulated genes classified as energy/ metabolism, six upregulated genes in the signal transduction category, two upregulated genes in the transcription/chromatin category, one upregulated gene in the structural protein category, three upregulated genes in the immune response category, three upregulated genes in the transport of nutrients/ions category, two upregulated genes in the stress/apoptosis category, and 11 upregulated genes in the other or unknown category (Table 3).
Table 2. Body weight and blood biochemical parameters in WKY and SHR/NDmc-cp rats.

\begin{tabular}{lcc}
\hline & WKY & SHRcp \\
\hline Body weight $(\mathrm{g})$ & $402 \pm 7.88$ & $603 \pm 23.00^{* *}$ \\
Plasma triglyceride $(\mathrm{mg} / \mathrm{dL})$ & $64 \pm 14.22$ & $946 \pm 230.36^{* *}$ \\
Plasma glucose $(\mathrm{mg} / \mathrm{dL})$ & $172 \pm 17.56$ & $326 \pm 99.91^{* *}$ \\
$\begin{array}{c}\text { Plasma insulin } \\
\quad(\mathrm{ng} / \mathrm{mL})\end{array}$ & $2.10 \pm 0.75$ & $14.01 \pm 0.29^{* *}$ \\
\end{tabular}

Values are indicated as mean \pm SD of 5-6 animals. ${ }^{* *} p<0.01$ by Student's $t$-test.

Real-time RT-PCR of the upregulated genes and PPAR protein expression in gastrocnemius muscles of the WKY and SHR/NDmc-cp rats

We performed real-time RT-PCR on the abovementioned upregulated genes in gastrocnemius muscles of the WKY and SHR/NDmc-cp rats. Among the 42 genes tested, the expression of 18 genes-Acox2, Asns, Acsm5, Pank2, Acot2, Psat1 and Nudt7 in the energy/metabolism category, RGD1560277, Mc5r and Pla2g7 in the signal transduction category, Atf5 in the transcription/ chromatin category, Acta2 in the structural protein category, Ifit 1 in the immune response category, Cat in the stress category, Ggta1p, Rtp4, RGD1309362 and RGD1306233 in the other or unknown category-was significantly higher in the SHR/NDmc-cp rats than in the WKY rats (Fig. 1A). The expression of $\operatorname{Rdh16}(p=0.051)$ in the energy/metabolism category, Pld5 $(p=0.084)$ in the signal transduction category, Slc7a5 $(p=0.060)$ in the transport of nutrients/ions category, and Cym $(p=0.061)$ in the other category tended to be higher in the SHR/NDmc-cp rats than in the WKY rats, but the difference was not significant (data not shown). The expression of other upregulated genes as determined by the microarray analysis did not differ between the two groups. We also tested the expression of the fatty acid oxidation-related genes, acyl-coenzyme A oxidase 1, palmitoyl $(A \operatorname{cox} 1)$, acyl-CoA synthetase long-chain family member 1 (Acsl1), Cpt1b, cytochrome c, somatic $(C y c s)$ and Ppargc 1a. The expression of Acox $1(p=0.059)$ and Acsl1 $(p=0.065)$ tended to be higher in the SHR/ NDmc-cp rats than in the WKY rats, but the difference was not significant (data not shown). Furthermore, the protein levels of ACOX2 were significantly higher in the SHR/NDmc-cp rats than in the WKY rats (Fig. 1B).

The gene expression of PPARs (Ppara, Ppard, Pparg1 and Pparg2) did not differ between the SHR/NDmc-cp rats and the WKY rats (data not shown). The protein level of PPARG1, but not PPARA, PPARD or PPARG2, was higher in the SHR/NDmc-cp rats than in the WKY rats (Fig. 1C).

Putative PPREs search in the upregulated genes

Next, we searched for putative PPREs with the websoftware PPREsearch $(16,17)$ in the upstream region ( $-5000 \mathrm{bp}$ to $-1 \mathrm{bp}$ ) of the 18 upregulated genes confirmed by real-time RT-PCR. We found putative PPREs in Acox2, Asns, Acsm5, Pank2, Psat1 and Nudt7 in the 


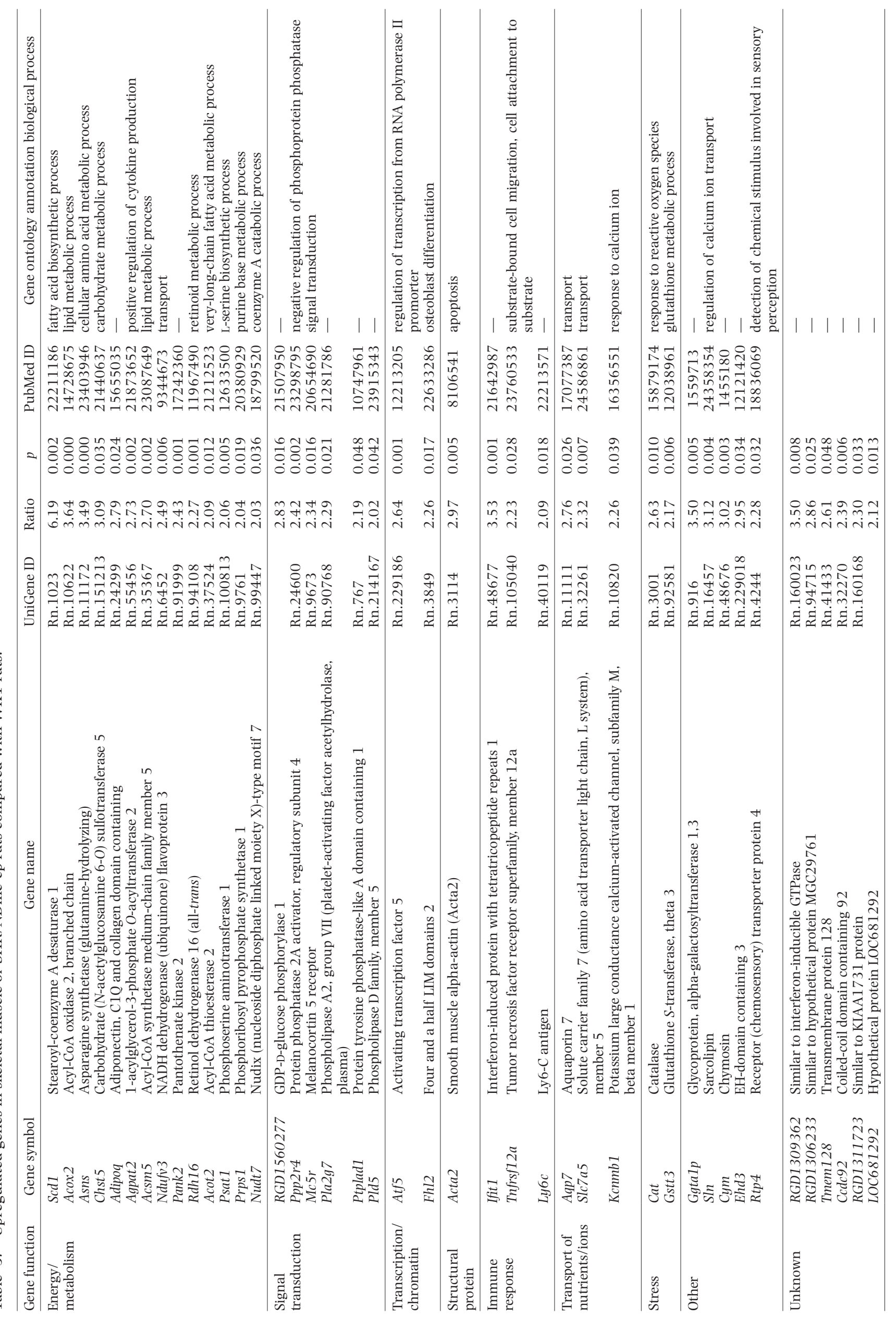


A
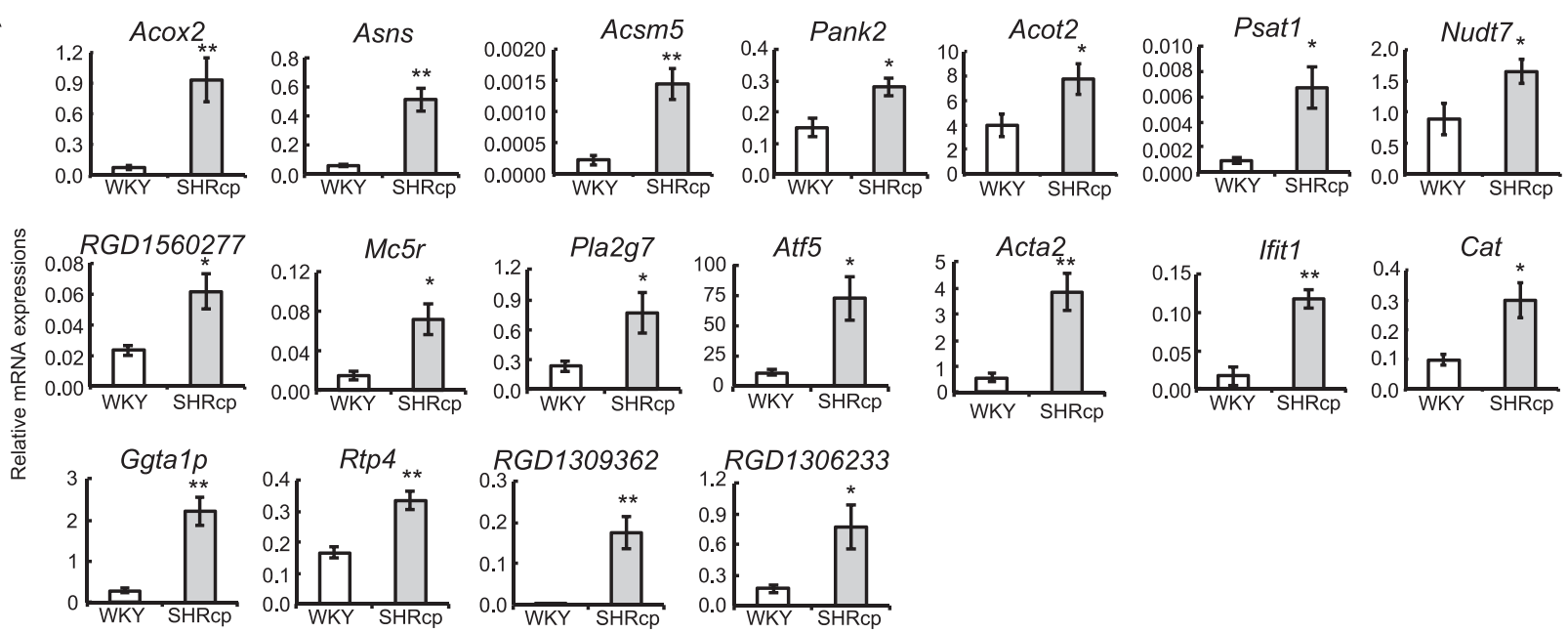

B

$\operatorname{ACOX} 2(77 \mathrm{kDa})$ A-TUBULIN (50kDa)
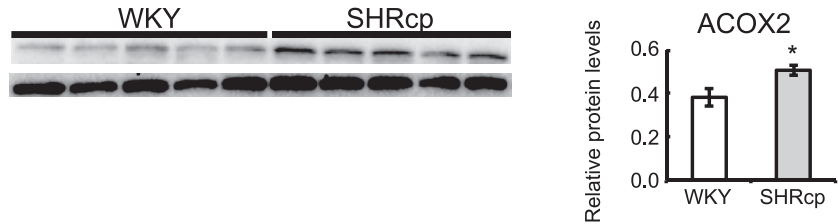

C
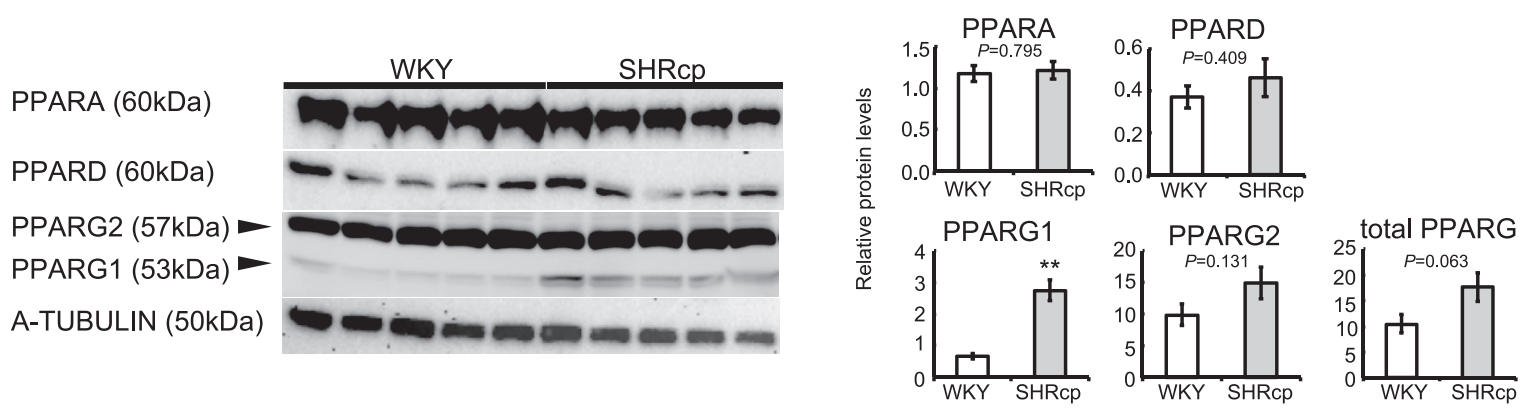

Fig. 1. RT-PCR of upregulated genes in microarray analysis and PPARs protein expression in gastrocnemius muscle. (A) mRNA levels of upregulated genes in microarray. Values were normalized against general transcription factor IIb (Gtf2b) $(n=5,6)$. (B) Protein levels of ACOX2. (C) Protein levels of PPARs. Quantification of protein levels was performed by normalization against A-TUBULIN $(n=5)$. Values are mean \pm SE. Statistical analysis was performed using a two-sided unpaired Student's $t$-test. ${ }^{* *} p<0.01,{ }^{*} p<0.05$, SHR/NDmc-cp (SHRcp) vs. WKY rats.

energy/metabolism category, RGD1560277 and Mc5r in the signal transduction category, Atf5 in the transcription/chromatin category, Acta2 in the structural protein category, Cat in the stress category, Ghta1p, Rtp4, RGD1306233 and RGD1309362 in the other or unknown categories (Table 4). Among the 18 upregulated genes confirmed by real-time RT-PCR, putative PPREs were found in the upstream region of 15 genes.

\section{DISCUSSION}

In this study, we found that the expression of many genes related to energy/metabolism, particularly fatty acid oxidation-related genes, such as Acox2, Asns, Acsm5, Pank2, Acot2, Psat1 and Nudt7, and other genes including Ifit1, Cat and Pla2g7, was higher in gastrocnemius muscle of the insulin-resistant MetS animal model, SHR/NDmc-cp rat, than in that of the normal model, WKY rat. In particular, we found that ACOX2 protein expression was significantly higher in the SHR/NDmccp rats than in the WKY rats. Our results demonstrate for the first time that the expression of genes related to fatty acid oxidation in the skeletal muscle is higher in the insulin-resistant MetS animals than in the control animals. It has been reported that $A \operatorname{cox} 2(18)$ and Nudt7 (19), Ifit1 (20), and Cat (21), are regulated by PPARs. In addition, it has been reported that the subtypes and/ or cascades of Acsm5 (18), Pank2 (22) and Acot2 (23) in the energy/metabolism category, and Pla2g7 (24) in the signal transduction category are targeted by PPARs. Furthermore, most genes ( 15 out of the 18 upregulated genes confirmed by real-time RT-PCR) possess putative PPREs in their promoter/enhancer region. Only in three genes, Acot2, Pla2g7, and Ifit1, did we not find putative PPREs in the promoter/enhancer region. However, as mentioned above, the expression of Ifit 1 and subtypes of $A \cot 2$ and Pla $2 g 7$ is enhanced by PPAR overexpression 


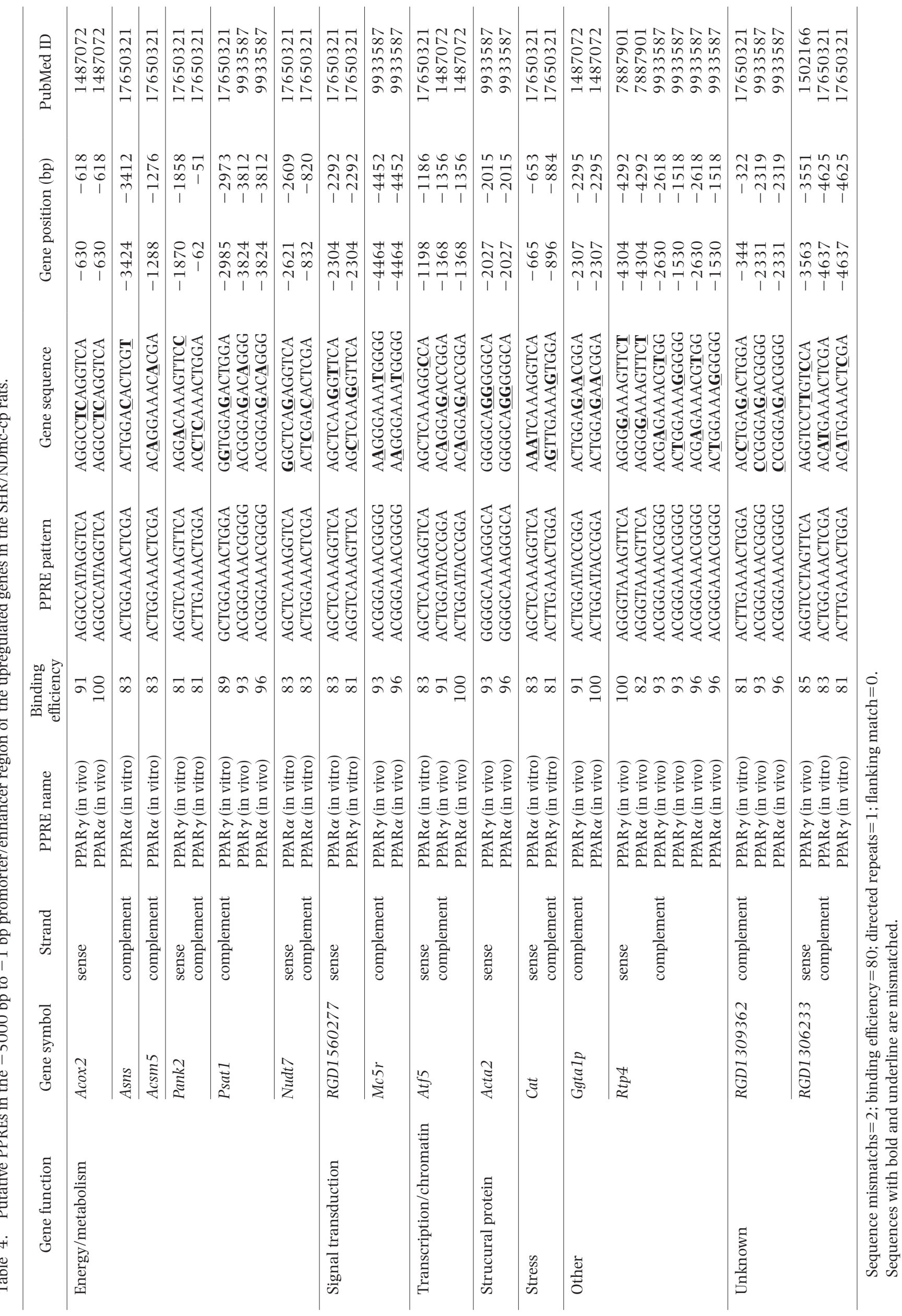


or PPAR agonist treatment. PPAR is one of the nuclear receptors that regulate fatty acid metabolism in each metabolic tissue and is activated by fatty acids and their metabolites (11). These results indicate that the expression of most genes in skeletal muscle induced by insulin resistance could be regulated by PPARs. It should be noted that we did not find reports on upregulation of genes possessing PPREs. We did not detect significant upregulation of the typical PPRE-possessing fatty acid oxidation-related gene, Acox 1 ( $p=0.059)$ (25), or of other typical fatty acid oxidation-related genes such as $\operatorname{Acsl1}(p=0.065)$ and $\operatorname{Cpt1b}(p=0.872)$. These results indicate that PPAR may target many genes broadly related to energy/metabolism, in particular genes related to fatty acid oxidation. Additionally, our results indicate that the upregulated genes rather than Acox 1 , Acsl1 and Cpt1b, may be strongly regulated by PPAR in skeletal muscle of SHR/NDmc-cp rats. However, this issue will be examined in further studies by chromatin immunoprecipitation (ChIP) assays. Additionally, it should be examined in future studies whether PPARs bind to the putative PPREs located in the promoter/ enhancer region of these genes in the gastrocnemius muscle of the SHR/NDmc-cp rats by ChIP assay, and whether PPARs transactivate these putative PPREs in skeletal muscle cell lines by reporter assays.

It is still unclear why other insulin-resistant models such as ZDF rats and C57BL/6J mice fed a high-fat diet did not show expression changes in fatty acid oxidationrelated genes (7-9). The pathology of the SHR/NDmc$\mathrm{cp}$ rats may have greater similarity to the human skeletal muscle with moderate insulin resistance than to ZDF rats and $\mathrm{C} 57 \mathrm{BL} / 6 \mathrm{~J}$ mice fed a high-fat diet. The skeletal muscle gene expression profiles should be compared between our current results and human skeletal muscle in various stages of insulin resistance, MetS and type 2 diabetes obtained by biopsy.

In this study, we did not detect significant differences in PPARA or PPARD protein expression between the SHR/NDmc-cp rats and the WKY rats. We showed that the protein expression of PPARG1, but not PPARG2, was higher in the SHR/NDmc-cp rats than in the WKY rats. Previously, it has been suggested that PPARG mainly functions in adipose tissues and macrophages (11). However, a recent study has demonstrated that musclespecific gene targeting of PPARG in mice induced fat accumulation in the adipose tissues and insulin resistance (26). Additionally, using in situ hybridization it has been shown that the expression of Pparg, whose isoforms were not divided in the experiment, was detectable in the skeletal muscle at a level of $5-10 \%$ of that in adipose tissues (27). This evidence indicates that PPARG functions in fatty acid oxidation and insulin sensitivity in the skeletal muscle. It has been reported that the expression of PPARG1, but not PPARG2, in human skeletal muscle obtained by a biopsy was positively associated with insulin resistance (28). Moreover, mouse muscle cell line C2C12 cells expressed Pparg1, but not Pparg2 (29). PPARG2 is mainly expressed in adipose tissues, and adipocytes are located in the perivascular space of the skeletal muscle, but not in the interstitium among muscle bundles, in SHR/NDmc-cp rats (13). Thus, PPARG1 expression in the skeletal muscle of SHR/ NDmc-cp rats may be associated with the expression of fatty acid oxidation-related genes and/or PPAR target genes. It should be examined in further studies whether PPARG1 is expressed in the skeletal muscle cells of SHR/ NDmc-cp rats, which are associated with the development of insulin resistance in rats. Here, we did not detect significant differences in the expression of Ppargc1a or $C p t 1 b$, which encode typical rate-controlling enzymes of fatty acid oxidation, between SHR/NDmc-cp rats and WKY rats. This may be because of differences in the responsiveness of subtypes or isoforms to each gene. To determine which PPAR subtypes/isoforms regulate the expression of each fatty acid oxidation-related gene, including Ppargc 1a and Cpt1b, in the skeletal muscle, in vivo ChIP assays should be performed.

In conclusion, our results show that insulin-resistant SHR/NDmc-cp rats, a MetS model, have a higher expression of a series of fatty acid oxidation-related genes, which are presumably targeted by PPARs, in skeletal muscle.

\section{Acknowledgments}

This work was supported by a Grant-in-Aid for Young Scientists (22680054) from the Ministry of Education, Culture, Sports, Science and Technology of Japan, and by the Takeda Science Foundation.

\section{REFERENCES}

1) DeFronzo RA. 1988. Lilly lecture 1987. The triumvirate: $\beta$-Cell, muscle, liver. A collusion responsible for NIDDM. Diabetes 37: 667-687.

2) Gorter PM, Olijhoek JK, van der Graaf Y, Algra A, Rabelink TJ, Visseren FL. 2004. Prevalence of the metabolic syndrome in patients with coronary heart disease, cerebrovascular disease, peripheral arterial disease or abdominal aortic aneurysm. Atherosclerosis 173: 363-369.

3) Watson RT, Pessin JE. 2001. Intracellular organization of insulin signaling and GLUT4 translocation. Recent Prog Horm Res 56: 175-193.

4) Long YC, Zierath JR. 2006. AMP-activated protein kinase signaling in metabolic regulation. J Clin Invest 116: $1776-1783$.

5) Alibegovic AC, Sonne MP, Højbjerre L, Bork-Jensen J, Jacobsen S, Nilsson E, Faerch K, Hiscock N, Mortensen B, Friedrichsen M, Stallnecht B, Dela F, Vaag A. 2010. Insulin resistance induced by physical inactivity is associated with multiple transcriptional changes in skeletal muscle in young men. Am J Physiol Endocrinol Metab 299: E752-763.

6) Jans A, Sparks LM, van Hees AM, Gjelstad IM, Tierney AC, Riserus U, Drevon CA, Roche HM, Schrauwen P, Blaak EE. 2011. Transcriptional metabolic inflexibility in skeletal muscle among individuals with increasing insulin resistance. Obesity (Silver Spring) 19: 2158-2166.

7) Voss MD, Beha A, Tennagels N, Tschank G, Herling AW, Quint M, Gerl M, Metz-Weidmann C, Haun G, Korn M. 2005. Gene expression profiling in skeletal muscle of Zucker diabetic fatty rats: implications for a role of stearoyl-CoA desaturase 1 in insulin resistance. Diabetologia 
48: 2622-2630.

8) Suh YH, Kim Y, Bang JH, Choi KS, Lee J W, Kim WH, Oh TJ, An S, Jung MH. 2005. Analysis of gene expression profiles in insulin-sensitive tissues from pre-diabetic and diabetic Zucker diabetic fatty rats. J Mol Endocrinol 34: 299-315.

9) de Wilde J, Smit E, Mohren R, Boekschoten MV, de Groot P, van den Berg SA, Bijland S, Voshol PJ, van Dijk KW, de Wit NW, Bunschoten A, Schaart G, Hulshof MF, Mariman EC. 2009. An 8-week high-fat diet induces obesity and insulin resistance with small changes in the muscle transcriptome of C57BL/6J mice. J Nutrigenet Nutrigenomics 2: 280-291.

10) Nangaku M, Miyata T, Sada T, Mizuno M, Inagi R, Ueda Y, Ishikawa N, Yuzawa H, Koike H, van Ypersele de Strihou C, Kurokawa K. 2003. Anti-hypertensive agents inhibit in vivo the formation of advanced glycation end products and improve renal damage in a type 2 diabetic nephropathy rat model. J Am Soc Nephrol 14: 1212-1222.

11) Grygiel-Gorniak B. 2014. Peroxisome proliferator-activated receptors and their ligands: nutritional and clinical implications-a review. Nutr J 13: 17.

12) Harikai N, Hashimoto A, Semma M, Ichikawa A. 2007. Characteristics of lipolysis in white adipose tissues of SHR/NDmc-cp rats, a model of metabolic syndrome. Metabolism 56: 847-855.

13) Hariya N, Mochizuki K, Inoue S, Morioka K, Shimada M, Okuda T, Goda T. 2014. Insulin resistance in SHR/ NDmc-cp rats correlates with enlarged perivascular adipcytes and endothelial cell dysfunction in skeletal muscle. J Nutr Sci Vitaminol 60: 52-59.

14) Chomczynski P, Sacchi N. 1987. Single-step method of RNA isolation by acid guanidinium thiocyanate-phenolchloroform extraction. Anal Biochem 162: 156-159.

15) Inamochi Y, Mochizuki K, Goda T. 2014. Histone code of genes induced by co-treatment with a glucocorticoid hormone agonist and a p44/42 MAPK inhibitor in human small intestinal Caco-2 cells. Biochim Biophys Acta 1840: 693-700.

16) Venkatachalam G, Sakharkar MK, Kumar AP, Clement MV. 2009. Peroxisome proliferator activator element search database. Int J Integr Biol 8(1): 37-42.

17) Venkatachalam G, Kumar AP, Yue LS, Pervaiz S, Clement MV, Sakharkar MK. 2009. Computational identification and experimental validation of PPRE motifs in NHE1 and MNSOD genes of human. BMC Genomics 10 (Suppl 3): S5.

18) Kim JH, Kim D, Kim J, Hwang JK. 2011. Euchresta horsfieldii benn. Activates peroxisome proliferator-activated receptor $\alpha$ and regulates expression of genes involved in fatty acid metabolism in human HepG2 cells. J Ethno- pharmacol 133: 244-247.

19) Reilly SJ, Tillander V, Ofman R, Alexson SE, Hunt MC. 2008. The nudix hydrolase 7 is an acyl-CoA diphosphatase involved in regulating peroxisomal coenzyme A homeostasis. J Biochem 144: 655-663.

20) Yu S, Matsusue K, Kashireddy P, Cao WQ, Yeldandi V, Yeldandi AV, Rao MS, Gonzalez FJ, Reddy JK. 2003. Adipocyte-specific gene expression and adipogenic steatosis in the mouse liver due to peroxisome proliferator-activated receptor $\gamma 1$ (PPAR $\gamma 1$ ) overexpression. J Biol Chem 278: 498-505.

21) Khoo NK, Hebbar S, Zhao W, Moore SA, Domann FE, Robbins ME. 2013. Differential activation of catalase expression and activity by PPAR agonists: implications for astrocyte protection in anti-glioma therapy. Redox Biol 1: 70-79.

22) Ramaswamy G, Karim MA, Murti KG, Jackowski S. 2004. PPAR $\alpha$ controls the intracellular coenzyme A concentration via regulation of PANK $1 \alpha$ gene expression. J Lipid Res 45: 17-31.

23) Dongol B, Shah Y, Kim I, Gonzalez FJ, Hunt MC. 2007. The acyl-CoA thioesterase I is regulated by $\operatorname{PPAR} \alpha$ and HNF $4 \alpha$ via a distal response element in the promoter. $J$ Lipid Res 48: 1781-1791.

24) Xu L, Han C, Lim K, Wu T. 2006. Cross-talk between peroxisome proliferator-activated receptor $\delta$ and cytosolic phospholipase $\mathrm{A}(2) \alpha /$ cyclooxygenase-2/prostaglandin $\mathrm{E}(2)$ signaling pathways in human hepatocellular carcinoma cells. Cancer Res 66: 11859-11868.

25) Ammerschlaeger M, Beigel J, Klein KU, Mueller SO. 2004. Characterization of the species-specificity of peroxisome proliferators in rat and human hepatocytes. Toxicol Sci 78: 229-240.

26) Hevener AL, He W, Barak Y, Le J, Bandyopadhyay G, Olson P, Wilkes J, Evans RM, Olefsky J. 2003. Musclespecific Pparg deletion causes insulin resistance. Nat Med 9: 1491-1497.

27) Braissant O, Foufelle F, Scotto C, Dauca M, Wahli W. 1996. Differential expression of peroxisome proliferator-activated receptors (PPARs): tissue distribution of PPAR- $\alpha,-\beta$, and $-\gamma$ in the adult rat. Endocrinology 137: $354-366$.

28) Kruszynska YT, Mukherjee R, Jow L, Dana S, Paterniti JR, Olefsky JM. 1998. Skeletal muscle peroxisome proliferator-activated receptor- $\gamma$ expression in obesity and non-insulin-dependent diabetes mellitus. J Clin Invest 101: 543-548.

29) Tsukahara T, Haniu H, Matsuda Y. 2013. Effect of alkyl glycerophosphate on the activation of peroxisome proliferator-activated receptor $\gamma$ and glucose uptake in $\mathrm{C} 2 \mathrm{C} 12$ cells. Biochem Biophys Res Commun 433: 281-285. 